\title{
A Non-Linear Controller for Forecasting the Rising Demand for Electric Vehicles Applicable to Indian Road Conditions
}

\author{
Poorani $\mathbf{S}^{1}$, Murugan $\mathbf{R}^{2}$ \\ ${ }^{1}$ Department of Electrical and Electronics Engineering, Karpagam University, Coimbatore, India \\ ${ }^{2}$ Department of Electrical and Electronics Engineering, Easwari Engineering College, Chennai, India
}

\begin{tabular}{l}
\hline Article Info \\
\hline Article history: \\
Received Apr 25, 2016 \\
Revised Jul 28, 2016 \\
Accepted Aug 16, 2016 \\
\hline
\end{tabular}

Keyword:

Artificial neural network

Electric vehicle

Load forecasting

Nonlinear controller

Training \& testing

\begin{abstract}
These days load forecasting is much more required in order to reduce the wastage of energy. This paper is to implement \& develop the idea of short term load forecasting by using Artificial Neural Network, the design of the neural network model, input data selection and Training \& Testing by using short term load forecasting will be described in paper. For the EV load forecasting only 2 variables are being used as temperature and humidity to forecast the output as load. This type of designed ANN model will be mapped by using historical data of temperature and humidity (taken from meteorological sites), whereas it is being Trained \& Tested by using historical data of loading of EV charging stations (Chetan maini ,Bangalore) of a particular area as Coimbatore to give the desired result. Training \& Testing done by using large amount of historical data of weather conditions and loading data $(\mathrm{kV})$. By the help of this model they can predict their daily loads (next day's load) by putting historical data in the acquired algorithm.
\end{abstract}

Copyright (C) 2016 Institute of Advanced Engineering and Science. All rights reserved.

\section{Corresponding Author:}

Poorani S,

Department of Electrical and Electronics Engineering,

Karpagam University,

Coimbatore.

Email: drpooranieee@gmail.com

\section{INTRODUCTION}

To calculate the almost accurate load forecasting it is necessary to go through the short term load forecasting [1],[2] for EV charging, load forecasting will be dependent upon the hourly load forecasting, the load can be forecasted up to 1 week ahead in hourly basis, but the main focus is to forecast the load for the recent next day (as tomorrow) which analyses \& decides the dispatcher power flow. Forecasting in power system is needed to prevent the power system \& power flow setup from any kind of damage and at the same time it reduces the wastage of energy [3],[4]. By seeing the graph of the loads it can be described and get the idea of load variation in hourly basis (for 24 hours), the analysis of peak load and load analysis as well is done. Power linear regression model has been developed that utilizes non-linear transformation \& other statically methodology to effectively capture the load variation due to special events, weather pattern, deviation in the other weather in 2 years of production use. It is to be stated that the performance of the algorithm will be acceptable only in the normal operating conditions so improvement in algorithm and variation is needed to enhance the accuracy in the performance in the rapid weather changing conditions or in a particular area where the weather will be changing rapidly in a short period. Few points to be considered while forecasting.

Input and output will be of completely non-linear relationship. model will not be very flexible for the rapid change in the environmental conditions or loading parameters $(\mathrm{kV})$. Recently there are being used more and more parameters in the place of 2 or 3 parameters [1] to calculate the forecasted load so that more precise \& accurate result can be acquired not only for the load forecasting but also in security purpose $\&$ fault 
diagnostic, neural network is being taken as helping tool because Tested \& Trained so that it can get the operators \& dispatchers confidence. Work has been done with the Chetan maini data. To train in such a way so that it can take the real time data of a particular place \& the output can be forecasted.

This paper presents the improved STLF modelling by taking temperature \& humidity as mapping parameters. First section of this paper are describing the basic neural network model to get the forecasted load in which load has been forecasted for the recent next day of ANN [5] model has been done that is called multi-layered feed forward neural network [3] for this model 72 inputs and 24 outputs are being taken. But since at the time of implementation only 24 neurons are being taken as inputs so there is a need to reduce 72 data (of 3 days in hourly basis) into 24 data (in hourly basis) by taking the mean of all 72 data (of 3 days) in the same hourly basis. In the neural network model large number of inputs will be used to reduce the weight update problems but then also the coefficients will be needed to update once or twice a year.

\section{INPUT VARIABLES}

72 inputs are being used along with the 24 hidden neurons \& 24 output neurons but in the used ANN model same number of neurons are being used, so it is need to reduce the 72 inputs into 24 inputs. Since all these 72 data are of 3 days (today, yesterday \& day before yesterday). So mean can be taken of all 3 days data in hourly basis to reduce the 72 data into 24 input data. In which weather conditions are being used for mapping \& loading data will be used for Training \& testing \& final forecasting. Performance of the input variables in the ANN model [6]. So the outputs can be of various kinds:

\subsection{Seasonal input load content}

Weather change and very less load will be changing, since loads will be changing slowly season to season [5]. It's all about cooling and heating loads over a year period when environmental conditions changes are being considered very less as compare to other tropical countries.

$\sin (2 \pi n . i / 365), \cos (2 \pi n . i / 365)$

where $n=(1,2,3)$

$\mathrm{i}$ ( $\mathrm{i}=1,2,-------365)$ number of days in an year.

\subsection{Weather condition input}

Temperature is most sensitive weather variable which affect the loading in the EV cars . If the area of the data calculation is being varied then weather coefficients for the forecasting will be different than one another because of their geographical and climatic conditions, so there will be somewhat different impact on the loading than the regular impact on the loading. There can be said that there are two types of temperature variables - direct and indirect. So direct temperature variable will work area to area whereas the temperature which affect the loading on the system level can be said as indirect temperature, in the EV level it is the temperature of the motor parts and other devices of the vehicle. So to consider the temperature of the vehicle temperature of all the devices should be taken care (that will also be in hourly basis). In the modelled diagram taking the environmental temperature into consideration there can be seen that how much temperature difference is there in the system.

It has been seen that there is a vast effect of temperature in the loading which means loading is very much sensitive to the temperature change. Nonlinear relationship has been seen between loading and temperature of the EV cars. A certain limit of humid climate increases the temperature and viseversa. Loading inputs are being used of Today, Yesterday and Day before yesterday, the loading data of early past is being used in this variable.

Other input variables can also be used [5] for mapping purpose and to give the loading data as input for the traing and testing as wind speed, cloud cover, dew point and it has been seen that they have very less effect on EV loading data (Chetan maini ). So other variables are being neglected to avoid the complexity in forecasting and to acquire an accurate result.

\section{NONLINEAR MODELING CRITERIA FOR LOAD FORECASTING AND TRAINING AND TESTING}

Nonlinear behaviour of the loading has been discussed earlier. Which is being influenced by the temperature and seasonal effects, this model is somewhat different than the earlier published papers because the main focus of the paper is to explain to explain the basic model of ANN based load forecasting system 
[7]. In the neural network load forecasting model when the output neurons are modelled with the nonlinear combination of the outputs of the hidden neurons. (2)

It can be given as follows :

$$
Y_{k}=w_{0}+H_{1} w_{11}+H_{2} w_{12}+H_{3} w_{13}+---------+H_{n} w_{1 n}
$$

where $H_{j}=H_{1}, H_{2},--------H_{n}$ is the output of the $j^{\text {th }}$ hidden neuron.

$w_{i j}=w_{0}, w_{11}, w_{12}-----------w_{i n}$ strength of signal from 1 neuron of a layer to all the neurons of the output layer. $w_{0}$ is the threshold output of the neuron $\mathrm{k}$.

From [1] the output of the hidden neuron can be grouped into 3 group: Non activated, Linear and Saturated. In both non activated and saturated neurons output will not very change according to the input change. whereas in the linear state the output will gradually change according to the input change, this is what is happening in the EV case, output will change by the change in the input.

So in the linear state will be seen in the description given below:

$$
\mathrm{H}_{\mathrm{j}}=\frac{1}{1+e^{\left(-\sum_{i=1}^{n} \mathrm{w}_{\mathrm{ij}}(\mathrm{n}) \cdot \mathrm{x}_{\mathrm{j}}(\mathrm{n})\right)}}
$$

where $X_{j}(n)$ is the input for the first layer of neuron model (after taking mean of the 3 days data).

$\mathrm{w}_{\mathrm{ij}}$ is strength of signal from 1 neuron of a layer to all the neurons of the output layer.

Note: Threshold state is being considered even though there will be negligible change in the output by changing the threshold state. So equation $1 \& 2$ shows that the output will be changing in the same order by the change in the input. So it can be said that its working in linear state and showing nonlinear behaviour .In the initial stages the linear terms are being taken only when the performance is somewhat deteriorated, because under these conditions load changes with the temperature and humidity in quadratic rate, that's why the non-linear input behaviour has been taken into account. So that the influence of cooling and heating degrees of the temperature can be seen in the load since the temperature is changing day to day in both the ends , so it is very essential to consider the each and every pint of temperature change so that actual effect of temperature can be seen in the loading.Then finally a set of trigonometric function has been taken to give the input in the Back propagation algorithm \& this model is modelled to provide 1 year behaviour of the loading.

\section{NEXT DAY'S LOAD MODELING}

So according to the standard modelling 3 days data as today, yesterday \& day before yesterday are being taken. In the place of 3 days data, many more days or may be less days than this can be taken as well but for our convenience we are taking 3 days input to forecast the next day's output. So historical loading data of previous days can be taken into consideration to calculate the forecasted data of the next day. Training \& Testing will be done by the historical data of the last year because for the comparison purpose actual data of the next day will be needing that is available in the previous year's historical data but the final forecasting data will be calculated of this year, since this year's real time data of forecasted day is not available so we need to acquire it by using mapping phenomenon, calculation is given below :

Let the hourly forecast of the next day is presented by:

$$
\left[H_{j}, \mathrm{j}=1,2,---------24\right] \text { and forecasting error }\left[\delta_{k}, \mathrm{k}=1,2,---------\mathrm{k} ; \mathrm{k}<24\right]
$$

Where $\mathrm{k}$ is the last hour of the next day with known hourly loads. So hourly load forecast is given by $\mathrm{k}$ that is $k^{\text {th }}$ hour.

$$
\delta_{k}=Y_{k}-t_{k} \quad ; \mathrm{k}=1,2,-------\mathrm{k}
$$

$t_{k}$ is real time loading data of the next day in $k^{\text {th }}$ hour.

Let the matrix contains variables $i$ and $j$. So the function of hourly loads is represented as:

$$
\text { \{fun (i, j), where } i=1,2,---------24 \text { and } j=1,2,---------24
$$

This matrix contains used historical loading data, this data is adjusted once in a year. So now the final forecasting has been done by the following formula: 


$$
Y_{k}=H_{j}+\text { fun }(\mathrm{i}, \mathrm{j}) \cdot \delta_{k}
$$

Where $\mathrm{j}=1$--------k, and $\mathrm{i}=\mathrm{k}+1$, ---------24

Training and Testing has been done with the real time historical data which substantially improve the ANN algorithm, which helps the dispatchers to send a particular required amount of energy.

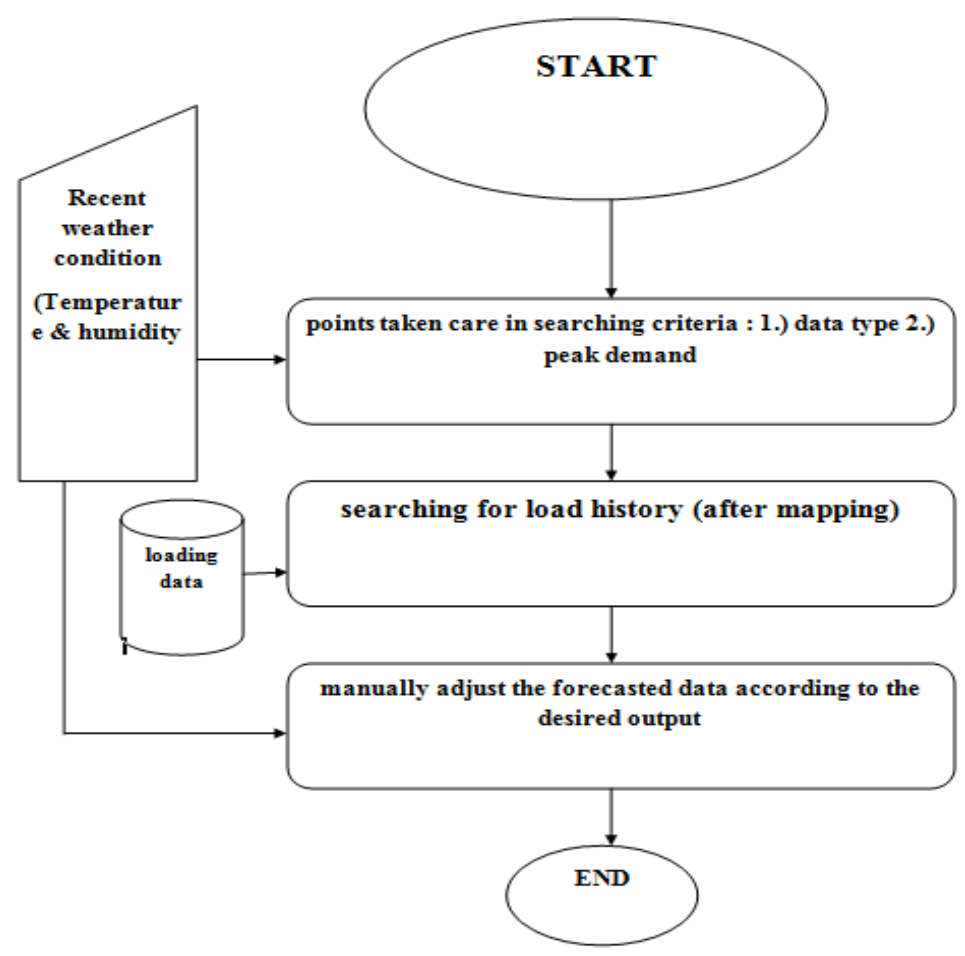

Figure 1. Equivalent block diagram for short term load forecasting

The following steps are being followed for the data processing:

\subsection{Mapping}

- 15 days data of temperature \& humidity of last year of any month (suppose August) and this year has been taken.

- Mapping is done with both years data and tried to acquire the loading data according to that.

- Mapping is done by taking temperature \& humidity data for finding the relation between loading data of this year and last year by using the temperature and humidity data of both of the years.

- Since we are working with the neural network so normalization of both set of loading data was needed that is previous year's (15days, same hours data) \& this year's (15days, same hour's data).

\subsection{Training \& Testing}

Training and testing from last year's data

- 3days loading data has been taken for Training \&Testing.

- Mean of all 3 days loading data in hourly basis is being calculate.

- After calculating mean and reducing the 72 data in 24 data Back propagation Algorithm [8] is being applied.

- Weights are being calculated by used Algorithm and then replaced assumed weight with calculated weight. 


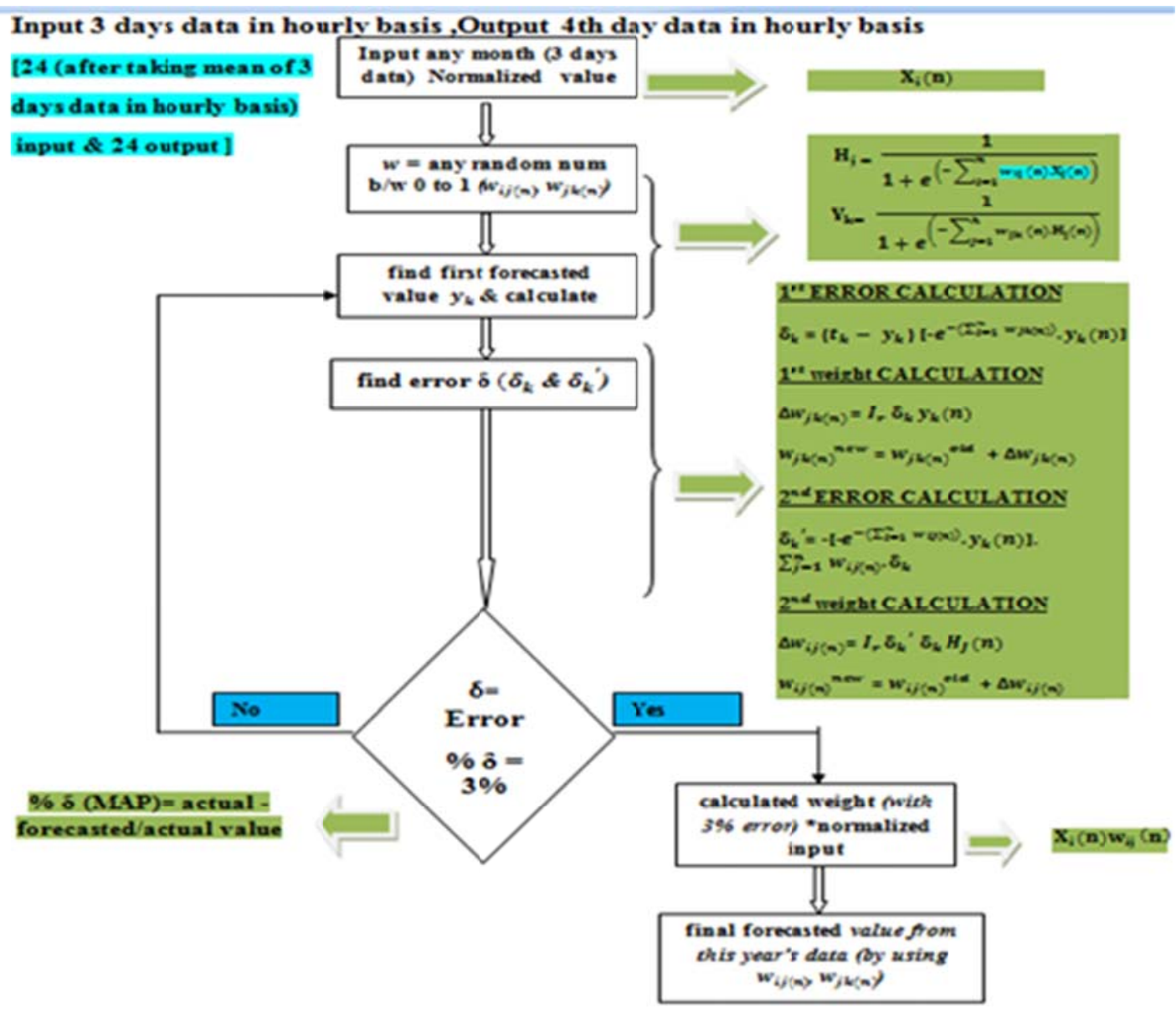

Figure 2. Algorithm flowchart

\subsection{Training \& testing from last year's data}

- For Training \& Testing 3days values of mapped normalized loading data are being taken, whereas 24 inputs and 24 outputs will be there in programming.

- This loading data is being trained in a particular model of ANN.

- Intermediate forecasted value has been found of that with assumed weight.

- $1^{\text {st }}$ and $2^{\text {nd }}$ error values and weighting values has been calculated and then by using those weighting values the forecasted data for the next day has been calculated.

\subsection{Last forecasted value with this year's data}

Intermediate forecasted value for next day with the trained weight has been found and then final forecasted value has been found.

\section{IMPLEMENTATION AND RESULT}

By taking data from (Chetan maini ) EV charging stations \& implementation has to bare multiple problems, which has been resolved. Most of the problems were regarding data acquiring and data communication, access the real time data from EV stations, man-man \& man-machine interfacing, downloading the data by using man-machine interfacing. Then after the collection of data there was another challenge to put the data in a certain and capable neural network model to give the desired result for the loading to forecast the next day's loading data by using historical loading data. Points to be considered at the time of implementation.

\subsection{On line \& off line implementation}

There will be 2 types of implementations by using neural network, the neural network [9] which is being used for Training \& Testing is called off line implementation. Here taking historical data (last year's 
data for input \& last year's real time data of forecasted day or next day to match with the last year's forecasted data were taken. Whereas in online implementation taking this year's real time data for input \& mapped (virtual) data for matching the mapped value. So this kind of implementation with the mapped data can be called as on line implementation. All the data will be Trained \& tested by using Back propagation algorithm by taking 3 days values in hourly basis, the online \& offline implementation will consist of one and one program. Offline implementation with the historical data \& online with the real time data.

\subsection{Simulation Result}

This section represent the comparison between on line \& offline implementation and the comparison between the real time data of the forecasted day \& real forecasted data for both online \& offline forecasted implementation which will help the dispatcher unit of Chetan maini, EV charging station. The results were obtained by using Back propagation algorithm.

Table 1.Training and Testing Stage

\begin{tabular}{cccc}
\hline \multirow{2}{*}{ S No } & \multicolumn{2}{c}{ Comparison between next day's real time data and forecasted data } \\
& Time & $\begin{array}{c}\text { Next } \begin{array}{c}\text { Year's Real Time } \\
\text { Data }\end{array} \\
\text { Result by Training and } \\
\text { Testing }\end{array}$ \\
\hline 1 & $00: 00: 00$ & 6.13 & 6.0926 \\
2 & $01: 00: 00$ & 5.24 & 5.201 \\
3 & $02: 00: 00$ & 5.69 & 5.6468 \\
4 & $03: 00: 00$ & 6.03 & 5.944 \\
5 & $04: 00: 00$ & 6.06 & 5.944 \\
6 & $05: 00: 00$ & 6.24 & 6.2412 \\
7 & $06: 00: 00$ & 8.38 & 8.4702 \\
8 & $07: 00: 00$ & 8.53 & 8.6188 \\
9 & $08: 00: 00$ & 9.37 & 9.5104 \\
10 & $09: 00: 00$ & 7.56 & 7.5786 \\
11 & $10: 00: 00$ & 11.07 & 11.2936 \\
12 & $11: 00: 00$ & 12.47 & 12.631 \\
13 & $12: 00: 00$ & 13.04 & 13.2254 \\
14 & $13: 00: 00$ & 14.86 & 15.1572 \\
15 & $14: 00: 00$ & 13.23 & 13.5226 \\
16 & $15: 00: 00$ & 8.1 & 8.173 \\
17 & $16: 00: 00$ & 10.94 & 11.145 \\
18 & $17: 00: 00$ & 9.85 & 9.956 \\
19 & $18: 00: 00$ & 11.48 & 11.590 \\
20 & $19: 00: 00$ & 11.66 & 11.888 \\
21 & $20: 00: 00$ & 10.14 & 10.253 \\
22 & $21: 00: 00$ & 12.35 & 12.631 \\
23 & $22: 00: 00$ & 8.9 & 8.916 \\
24 & $23: 00: 00$ & 7.98 & 8.024 \\
\hline & & & \\
& & & \\
\hline
\end{tabular}

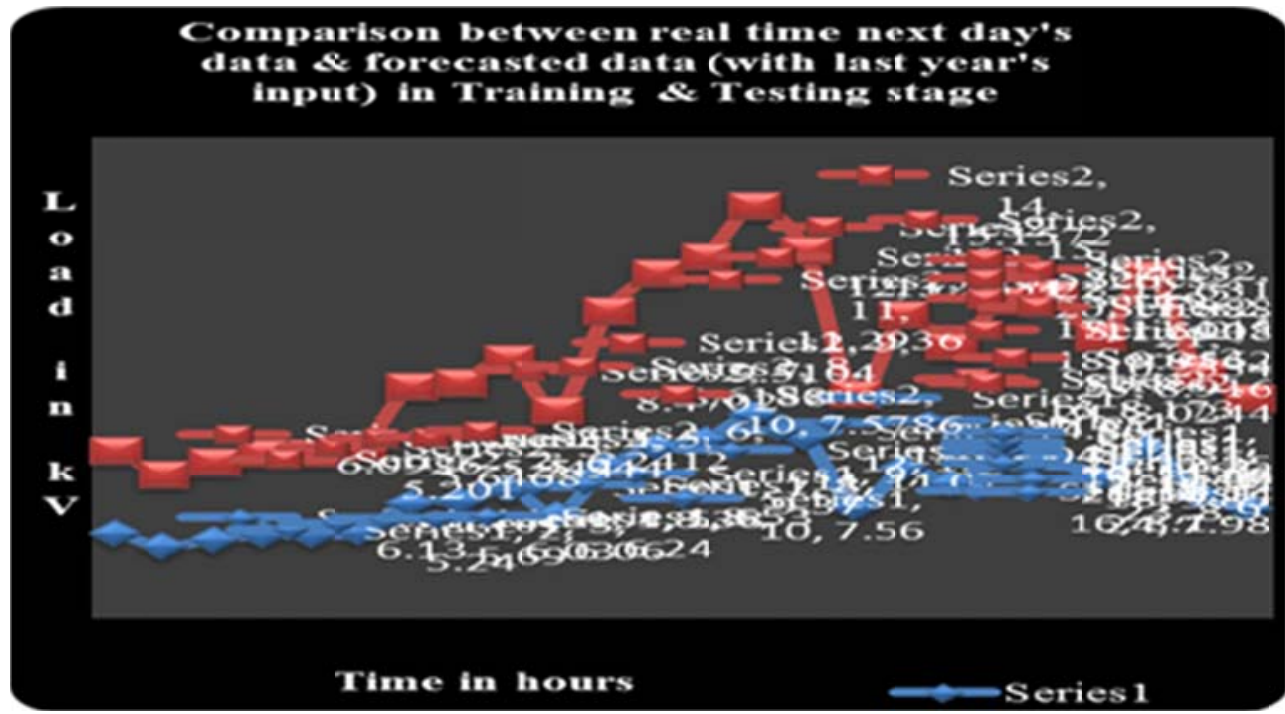

Figure 3. Training and testing reult with real time value of loading (kV) of Feb 2016 (Red line- Real time data of next day. Blue line- Forecasted data of next day)

A Non Linear Controller for Forecasting the Rising Demand for Electric Vehicles Applicable .... (Poorani S) 
Table 2. Comparison between mapped data with this year's input

\begin{tabular}{cccc}
\hline S .No & \multicolumn{2}{c}{ Comparison between mapped data with this year's input } \\
& Time & $\begin{array}{c}\text { Next Year's Real } \\
\text { Time Data }\end{array}$ & $\begin{array}{c}\text { Result By Training } \\
\text { And Testing }\end{array}$ \\
\hline 1 & $00: 00: 00$ & 5.5 & 5.376 \\
2 & $01: 00: 00$ & 5 & 4.872 \\
3 & $02: 00: 00$ & 4.6 & 4.368 \\
4 & $03: 00: 00$ & 4.77 & 4.536 \\
5 & $04: 00: 00$ & 5.5 & 5.376 \\
6 & $05: 00: 00$ & 7.2 & 7.224 \\
7 & $06: 00: 00$ & 10.4 & 10.416 \\
8 & $07: 00: 00$ & 12.4 & 12.6 \\
9 & $08: 00: 00$ & 11.5 & 11.592 \\
10 & $09: 00: 00$ & 8.5 & 8.568 \\
11 & $10: 00: 00$ & 8.8 & 8.736 \\
12 & $11: 00: 00$ & 9 & 9.072 \\
13 & $12: 00: 00$ & 11.5 & 11.592 \\
14 & $13: 00: 00$ & 15 & 15.288 \\
15 & $14: 00: 00$ & 16 & 16.296 \\
16 & $15: 00: 00$ & 14.4 & 14.614 \\
17 & $16: 00: 00$ & 11.5 & 11.592 \\
18 & $17: 00: 00$ & 16.8 & 17.136 \\
19 & $18: 00: 00$ & 15.4 & 15.792 \\
20 & $19: 00: 00$ & 12.4 & 12.6 \\
21 & $20: 00: 00$ & 11 & 11.088 \\
22 & $21: 00: 00$ & 13.5 & 13.776 \\
23 & $22: 00: 00$ & 11 & 11.088 \\
24 & $23: 00: 00$ & 8 & 8.064 \\
\hline
\end{tabular}

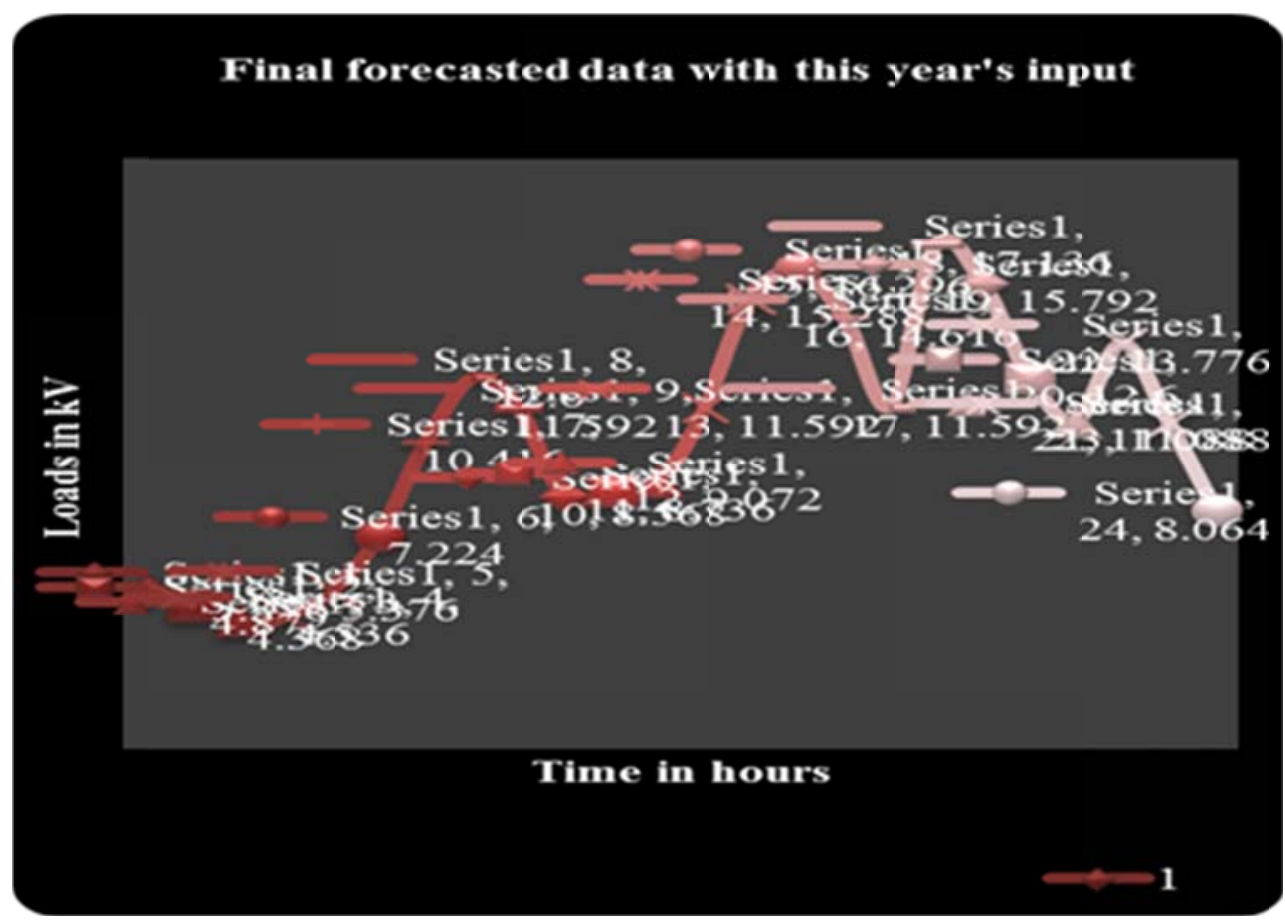

Figure 4. Final forecasted data with real time value of loading (kV) of August 2016 (Red line- Final forecasted data of next day)

\section{DISCUSSION}

In the literature survey done by certain authors it has been stated that the use of data mining methods for forecasting the EV charging demand was studied. Two different realistic study case where considered and the performance of four different data mining methods was evaluated. In the first study case the day-ahead charging demand of 3,000 EV was forecasted and compared with the actual data. The second study case considered a fleet of 2,130 EV and predicted the charging demand of whole week on a half-hourly basis. The 
results showed that the data mining methods can be used for forecasting the EV charging load, with increased accuracy especially when the configuration parameters of each method are carefully selected.

However the results of the final forecasting [10] \& Training \& Testing will be almost same, there won't be much difference only the difference will be of data because that are using last year's data for Training \& Testing \& moreover this year's data for final forecasting as input but the models that are being used for both the forecasting will be same that's why there won't be much difference in both the results. The new STLF algorithm will come with the following advances.

The main problem with the statistical model with almost be some experimental error while implementing the result. So to overcome this error and give almost accurately precise result we work with the neural network which will deal with the real time data \& give the desired result with less amount of error compared with the other statistical techniques. Neural network is particularly effective in handling outliers, although other methods also can make it done at some extent. The input that is being selected by the mapping process before the final forecasting, since for the final forecasting the real time data is not available of the final forecasted year that's why we need to acquire it by mapping process that is hit \& trail method that will be based on previous experiences [4]. Temperature \& humidity modeling is being done for the mapping process to get the loading data for the next year so that we can use the data for the matching process for the real forecasted data. Temperature and humidity will give the mapped loading data that will be used for the comparison with the forecasted data of this year so that we can encounter the error of the final forecasted data by seeing the mapped data (mapped data : approximate of the real time data).

\section{REFERENCES}

[1] D. Park, et al., "Electric Load Forecasting Using an ArtificialNeural Network," IEEE Trans. on Power Systems, vol/issue: 6(2), pp. 442-449. 1991.

[2] Z. Xinbo and C. Jinsai, "Short-term power system load forecasting based on improved BP artificial neural network," Computer Science and Automation Engineering (CSAE), 2011 IEEE International Conference on, Shanghai, pp. 14-17, 2011.

[3] J. M. Espinoza, et al., "Electric Load Forecasting,” IEEE Control Systems, vol/issue: 27(5), pp. 43-57, 2007.

[4] S. Vemuri, et al., "On-Line Algorithms for Forecasting Hourly Loads of an Electric Utility," IEEE Trans. Power App. \& Syst, vol/issue: PAS-100(8), pp. 3775-3784, 1981.

[5] A. D. Papalexopoulos, et al., "An implementation of a neural network based load forecasting model for the EMS," IEEE Transactions on Power Systems, vol/issue: 9(4), 1994.

[6] Webberley and D. W. Gao, "Study of artificial neural network based short term load forecasting," Power and Energy Society General Meeting (PES), 2013 IEEE, Vancouver, BC, pp. 1-4, 2013.

[7] Jain and B. Satish, "Clustering based Short Term Load Forecasting using Artificial Neural Network," Power Systems Conference and Exposition, 2009. PSCE '09. IEEE/PES, Seattle, WA, pp. 1-7, 2009.

[8] Azriyenni, et al., "Backpropogation Neural Network Modeling for Fault location in Transmission Line $150 \mathrm{KV}$," Indonesian Journal of Electrical Engineering and Informatics (IJEEI), vol/issue: 2(1), pp. 1-12, 2014.

[9] N. Yousefi, "A New Hybrid Wavelet Neural Network and interactive Honey Bee Matting Optimization based on islanding Detection,” Indonesian Journal of Electrical Engineering and Informatics (IJEEI), vol/issue: 3(4), pp. 224-238, 2015.

[10] P. Sharma, "NARMA-L2 Controller for five-Area load Frequency control,” Indonesian Journal of Electrical Engineering and Informatics (IJEEI), vol/issue: 2(4), pp. 170-179, 2014. 